\title{
Stromal Progenitor Cells in Bone Marrow of Patients with Aplastic Anemia *
}

\author{
E. Elstner, E. Schulze, R. Ihle, H. Stobbe, and S. Grunze
}

\section{A. Introduction}

The pathogenesis of aplastic anemia (AA), which is defined by pancytopenia and hypocellularity of the bone marrow (BM), is still an unsolved problem and is most probably heterogeneous. Hemopoietic stromal defects have been suggested as one of the possible pathophysiologic mechanisms for AA. Up to now, in all investigations on stromal cells of patients with AA, the fibroblast colony assay (CFU-F) has been used $[4,12,13]$. Fibroblasts, however, are only one component of the hemopoietic stroma; they are not capable of maintaining hemopoiesis in vitro alone.

For our study we used the Dexter culture [1] modified for human BM [3], because in this system a complex adherent cell layer develops after 2 weeks, containing, in addition to fibroblasts, other stromal elements (macrophages, adipocytes, endothelial cells) and which is known to maintain hemopoiesis in this system for several weeks. The aim of these investigations was to find out if there are differences in the ability to form stromal elements between normal and AA BM cells in vitro.

\section{B. Material and Methods}

BM of nine normal volunteers and six patients with idiopathically acquired AA

* University Hospital (Charité), Department of Internal Medicine, Division of Hematology, Berlin, GDR, and Central Institute of Molecular Biology of the Academy of Sciences of the GDR, Berlin, GDR
(Table 1) were investigated in a liquid system [3] with slight modifications (Fig. 1). The BM cells were obtained by iliac crest puncture $(2 \mathrm{ml})$ and immediately diluted with the same volume of McCoy's 5A medium (Serva) containing heparin without preservative (Gedeon Richter, Hungary). After spontaneous sedimentation of erythrocytes, the supernatant, including the fat droplets floating on the surface (the lipid substance can be utilized as a source of energy in rapidly proliferating cell systems) [11], was added to the culture medium (McCoy's 5A supplemented with $10 \%$ fetal calf serum, SIFIN, GDR) and $10 \%$ horse serum (Flow, Manchester) in order to get a cell concentration of $5 \times 10^{5}$ cells per milliliter suspension. The cells were cultivated in Petri dishes at $37^{\circ} \mathrm{C}$ in $7.5 \% \mathrm{CO}_{2}$ for 14 days without feeding. After 14 days, the liquid fraction was removed. The air-dried adherent layer was stained according to Pappenheim. GM-CFC of 18 AA patients was performed in a double-layer culture (Pike and Robinson) [10].

Table 1. Criteria for diagnosis of aplastic anemia

Aplastic anemia is defined by:

Pancytopenia

Hypocellularity of the hemopoietic bone marrow, with absence of neoplastic infiltration or significant fibrosis

It is distinct from:

Aplasia induced by cytostatics or irradiation Primary pancytopenia with hyperplastic marrow or myelodysplasia 


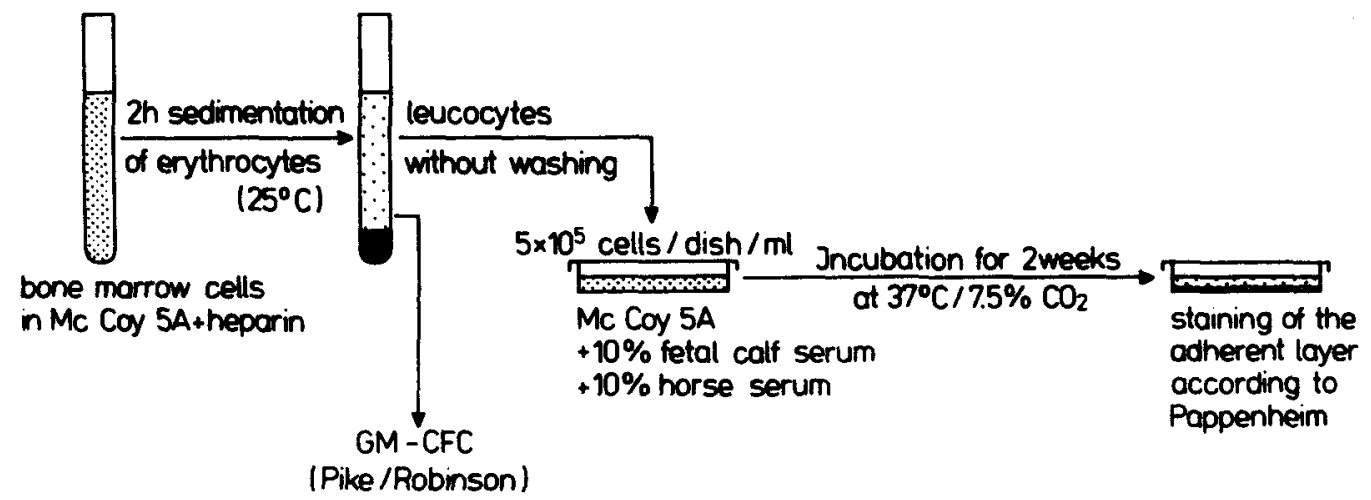

Fig. 1. Schematic representation of the method

\section{Results}

Figure 2 shows the growth pattern of GMCFC of BM from patients with AA in agar culture. The number of colonies $(>40$ cells) was decreased in most cases. The results of the stromal cultures are represented in Fig. 3. After 1 week of cultivation, there was a very poor adherent layer in both normal and aplastic BM cultures, and no difference between them could be detected. After 14 days, however, an adherent layer was established, which was distinctly more compact in the BM cultures of normal subjects compared with those of patients with AA (Figs. 4, 5). In addition to these quantitative differences, qualitative differences were also apparent. Thus, in normal BM cultures well-established "cobblestone" areas were formed (Fig. 4). In cultures of AA BM, such areas were rare.

\section{Discussion}

The pancytopenia and hypocellularity of the hemopoietic $\mathrm{BM}$ in $\mathrm{AA}$ patients is the final outcome of functional failure in the hemopoietic stem cells (HSC). In most AA patients, the number of GM-CFC, BFU-E, CFU-E, CFU-D, and CFU-mix is decreased $[2,5,7,9]$. For effective hemopoiesis it is essential to have an intact microenvironment (stroma cells), on which HSC renew and differentiate. Therefore, the failure of the HSC in AA can also be caused secondarily by damage of the stromal cells, as was shown in experimentally induced aplasia in animals $[6,8]$. The

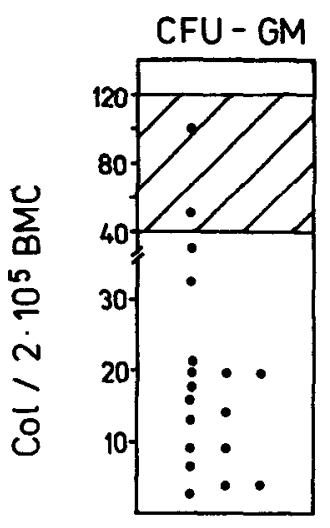

Fig. 2. Growth pattern of bone marrow from patients with aplastic anemia in agar culture (hatched area shows range of 20 normal volunteers)

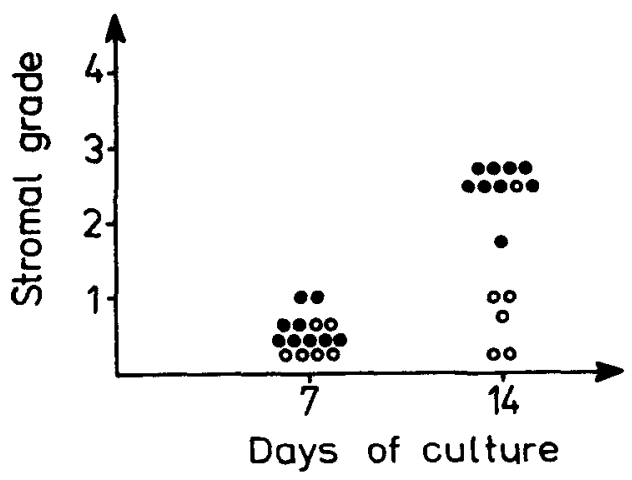

Fig. 3. Stromal layer in liquid culture from bone marrow cells. Full circles normal volunteers; open circles patients with aplastic anemia. Each dish was assigned a score from 1 to 4 , corresponding to a stromal layer covering from $25 \%$ to $100 \%$ of the area of the culture dish

pathogenetic role of stromal cells in the development of AA in vitro has been investigated up to now only with CFU-F assay, which gives evidence for only one component of stromal cells, i.e. fibroblasts, and no information about the interplay of stromal cells and HSC. For our investi- 

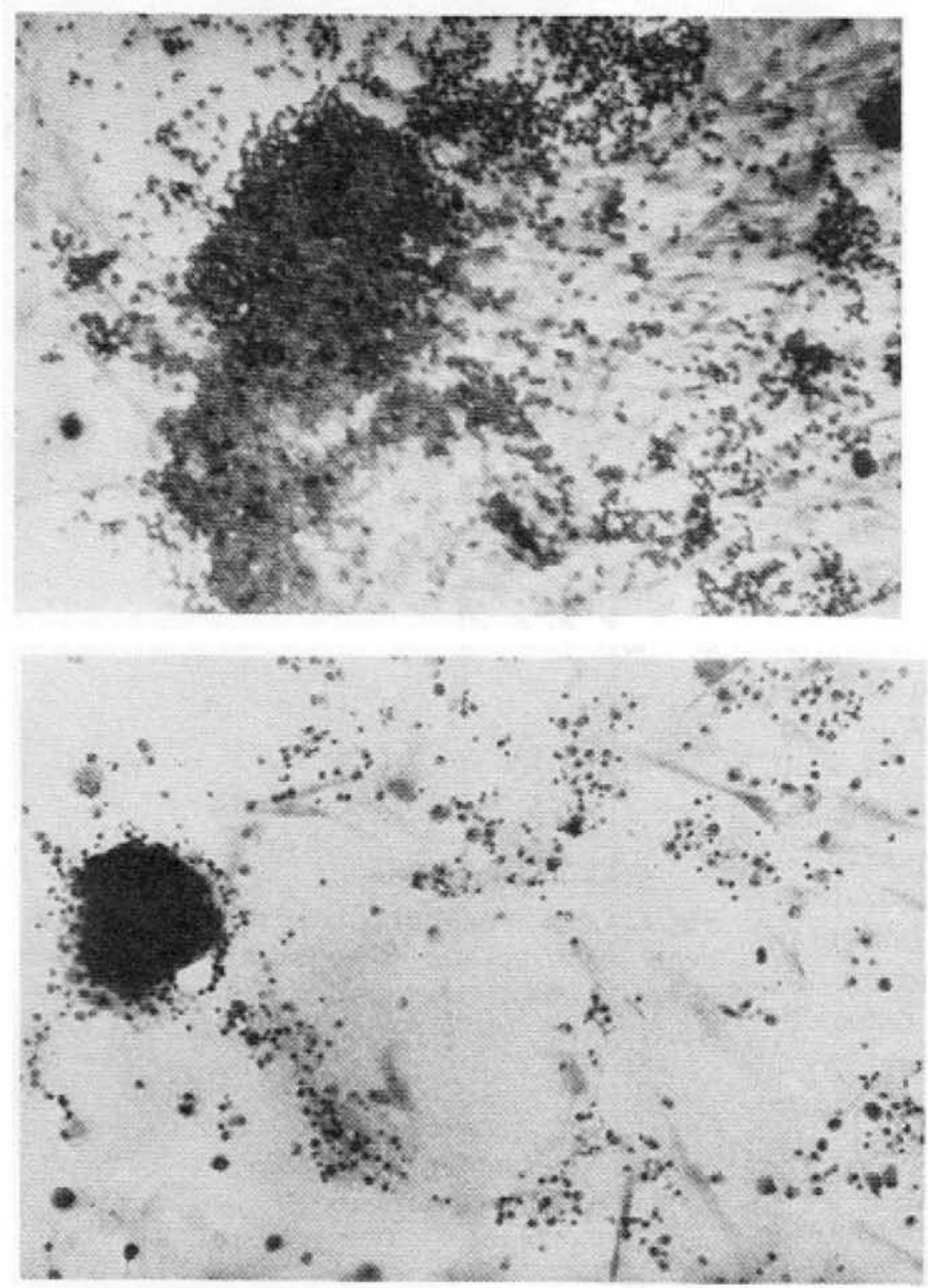

Fig. 4. Pappenheim-stained adherent layer with hemopoietic islands formed by normal bone marrow after 14 days cultivation $(\times 32)$
Fig. 5. Pappenheim-stained adherent layer formed by aplastic anemia bone marrow after 14 days cultivation $(\times 32)$ gations we have used the Dexter culture [3] which allows study of the interaction between the complex stroma and hemopoiesis.

Our findings indicate that in AA not only is hemopoiesis insufficient, but the stromal cells are affected also. It is not clear whether these two phenomena are independent of each other, or if the decreased number of HSC is caused by a disturbed microenvironment. Since for our experiments we used unwashed cells, including the patients' sera, autoimmunologic processes (humoral and cellular) cannot be excluded.

\section{References}

1. Dexter TM, Allen TD, Lajtha LG (1977) Conditions controlling the proliferation of haemopoietic stem cells in vitro. J Cell Physiol 91:335-344

2. Elstner E, Schulze E, Ihle R, Schütt $M$, Stobbe H (1983) Proliferation and maturation of hemopoietic cells from patients with preleukemia and aplastic anemia in agar and diffusion chamber cultures. In: Neth, Gallo, Greves, Moore, Winkler (eds) Haematology and blood transfusion, vol 28, Modern trends of human leukemia V. Springer, Berlin Heidelberg, pp 358-361

3. Gartner S, Kaplan HS (1980) Long-term culture of human bone marrow cells. Proc Natl Acad Sci USA 77:4756-4759

4. Gordon MY, Gordon-Smith EC (1981) Bone marrow fibroblastoid colony-forming cells (P-CFC) in aplastic anaemia: colony growth and stimulation of granulocyte-macrophage colony-forming cells (GM-CFC). Br J Haematol 49:465-477

5. Hara H, Kai S, Fushimi M, Taniwaki S, Okomoto T, Ohe Y, Fujita S, Noguchi K, 
Senba M, Hamane M, Kanamaru A, Nagai K (1980) Pluripotent hemopoietic precursors in vitro $\left(\mathrm{CFU}_{\mathrm{mix}}\right)$ in aplastic anemia. Exp Hematol 8: 1165-1171

6. Hays E, Hale L, Villarreal B, Fitchen JM (1982) "Stromal" and hemopoietic stem cell abnormalities in long-term cultures of marrow from busulfan-treated mice. Exp Hematol 10:383-392

7. Heimpel H, Heit W (1983) Panmyelophthisis and other bone marrow disorders. Verh Dtsch Ges Pathol 67:276-285

8. Knospe WH, Blom J, Crosby WH (1968) Regeneration of locally irradiated bone marrow I. Dose dependent long-term changes in the rat with particular emphasis upon vascular and stromal reactions. Blood 28:398

9. Kurnick JE, Robinson WA, Dickey CA (1971) In vitro granulocytic colony forming potential of bone marrow from patients with granulocytopenia and aplastic anemia. Proc Soc Exp Biol Med 137:917-920
10. Pike BL, Robinson WA (1970) Human bone marrow colony growth in agar-gel. J Cell Physiol 76:77-84

11. Tavassoli M (1984) Marrow adipose cells and hemopoiesis: an interpretative review. Exp Hematol 12:139-146

12. Wiktor-Jedrzejczak W, Siekierzynski M, Szczylik C, Gornas P, Dryjanski T (1982) Aplastic anemia with marrow defective information of fibroblastoid cell colonies in vitro. Scand J Haematol 28: 82-90

13. Zaritsky AY, Kulik MA, Kazhdan IJ, Lisovskaya VA, Ivasenko IN, Arkadyeva GE (1983) Colony-forming capacity of fibroblast precursor cells of the bone marrow in patients with different neutropenic conditions. Ter Arkh 8:110-114 